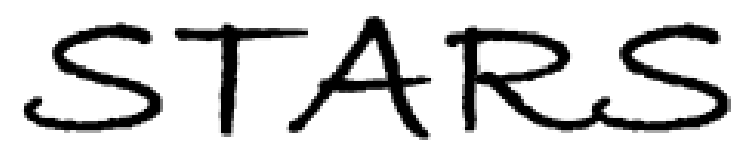

University of Central Florida

STARS

$1-1-2009$

\title{
Multiplexed highly sensitive detections of cancer biomarkers in thermal space using encapsulated phase change nanoparticles
}

Liyuan Ma

University of Central Florida

Yan Hong

University of Central Florida

Zeyu Ma

University of Central Florida

Charalambos Kaittanis

University of Central Florida

J. Manuel Perez

University of Central Florida

Find similar works at: https://stars.library.ucf.edu/facultybib2000

University of Central Florida Libraries http://library.ucf.edu

See next page for additional authors

This Article is brought to you for free and open access by the Faculty Bibliography at STARS. It has been accepted for inclusion in Faculty Bibliography 2000s by an authorized administrator of STARS. For more information, please contactSTARS@ucf.edu.

\section{Recommended Citation}

Ma, Liyuan; Hong, Yan; Ma, Zeyu; Kaittanis, Charalambos; Perez, J. Manuel; and Su, Ming, "Multiplexed highly sensitive detections of cancer biomarkers in thermal space using encapsulated phase change nanoparticles" (2009). Faculty Bibliography 2000s. 1847.

https://stars.library.ucf.edu/facultybib2000/1847

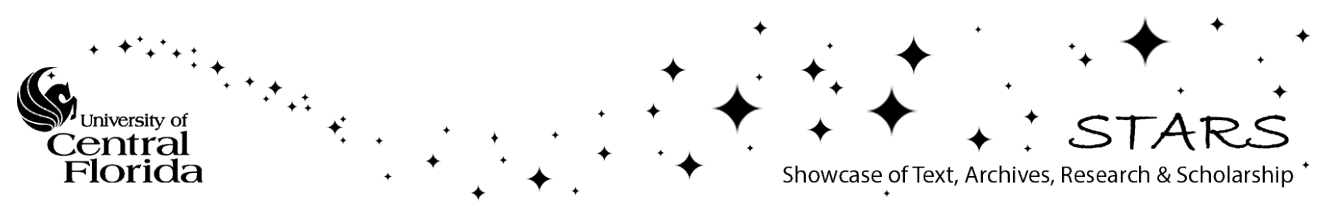




\section{Authors}

Liyuan Ma, Yan Hong, Zeyu Ma, Charalambos Kaittanis, J. Manuel Perez, and Ming Su 


\section{Multiplexed highly sensitive detections of cancer biomarkers in thermal space using encapsulated phase change nanoparticles}

Cite as: Appl. Phys. Lett. 95, 043701 (2009); https://doi.org/10.1063/1.3189082

Submitted: 21 May 2009 . Accepted: 28 June 2009 . Published Online: 27 July 2009

Liyuan Ma, Yan Hong, Zeyu Ma, Charalambos Kaittanis, J. Manuel Perez, and Ming Su

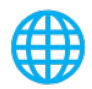

\section{ARTICLES YOU MAY BE INTERESTED IN}

Encoding and decoding nanoscale thermal barcodes for ultrahigh capacity identification systems

Applied Physics Letters 95, 233101 (2009); https://doi.org/10.1063/1.3271524

Multiplexed biomarker detection using x-ray fluorescence of composition-encoded nanoparticles

Applied Physics Letters 97, 263704 (2010); https://doi.org/10.1063/1.3533817

\section{Applied Physics Letters}

Mid-IR and $\mathrm{THz}$ frequency combs special collection 


\title{
Multiplexed highly sensitive detections of cancer biomarkers in thermal space using encapsulated phase change nanoparticles
}

\author{
Liyuan Ma, ${ }^{1}$ Yan Hong, ${ }^{1,2}$ Zeyu Ma, ${ }^{1,2}$ Charalambos Kaittanis, ${ }^{1,3}$ J. Manuel Perez, ${ }^{1,4}$ and \\ Ming Su, ${ }^{1,2, a)}$ \\ ${ }_{1}^{1}$ NanoScience Technology Center, University of Central Florida, Orlando, Florida 32826, USA \\ ${ }^{2}$ Department of Mechanical, Materials, and Aerospace Engineering, University of Central Florida, Orlando, \\ Florida 32826, USA \\ ${ }^{3}$ Biomolecular Science Center, University of Central Florida, Orlando, Florida 32826, USA \\ ${ }^{4}$ Department of Chemistry, University of Central Florida, Orlando, Florida 32826, USA
}

(Received 21 May 2009; accepted 28 June 2009; published online 27 July 2009)

\begin{abstract}
We describe a multiplexed highly sensitive method to detect cancer biomarkers using silica encapsulated phase change nanoparticles as thermal barcodes. During phase changes, nanoparticles absorb heat energy without much temperature rise and show sharp melting peaks $\left(0.6{ }^{\circ} \mathrm{C}\right)$. A series of phase change nanoparticles of metals or alloys can be synthesized in such a way that they melt between 100 and $700{ }^{\circ} \mathrm{C}$, thus the multiplicity could reach 1000 . The method has high sensitivity $(8 \mathrm{nM})$ that can be enhanced using materials with large latent heat, nanoparticles with large diameter, or reducing the grafting density of biomolecules on nanoparticles. (C) 2009 American Institute of Physics. [DOI: 10.1063/1.3189082]
\end{abstract}

A major challenge of biomarker-based early detection of cancer is that some biomarkers (nucleic acids or proteins) exist at low concentration and are not effective in reflecting cancers. ${ }^{1}$ Multiplexed highly sensitive detection of multiple biomarkers has been proposed to enhance the accuracy. ${ }^{2}$ Nanoparticles with unique magnetic, electric, optical, and electrochemical signatures have been used to detect biomarkers with high sensitivity by converting biological events into physical signals that can be amplified, ${ }^{3-6}$ but the detection multiplicity is limited due to narrow detection range and wide peak. ${ }^{7}$ Only one or few types of biomarkers can be detected each time, and screening one sample for multiple biomarkers takes a long time. Magnetic nanoparticles lack particle-specific property for multiplicity. ${ }^{8}$ Electric measurement cannot distinguish two types of metallic nanoparticles. ${ }^{9}$ Although few types of nanoparticles can be detected by electrochemical method, ${ }^{10}$ the method spans several volts, and the peaks are too broad to offer large multiplicity. The absorbance peaks of optically active nanoparticles in the UVvisible region (400-900 $\mathrm{nm}$ ) are broad with peak width at half height of $150 \mathrm{~nm},{ }^{11-14}$ which limits the detection capacity. In case of surface enhanced Raman spectroscopy where sharp peaks exist over a large range, the available Raman active dyes are limited, ${ }^{15}$ the ratio of Raman "hot" nanoparticles is low, and quantitative signals are hard to obtain.

The solid to liquid phase change materials (PCM) have unique thermophysical properties. PCM can adsorb thermal energy without temperature rise during the melting process. As long as the solid phase exists, all adsorbed thermal energy is used to melt the solid phase, and the theoretical peak width is zero. ${ }^{16}$ In practice, the heat transfer from the surrounding environment into the core of PCM takes time, and such delay in melting broadens the melting peak because the temperature scan is done at a constant rate $\left(0.02-20{ }^{\circ} \mathrm{C} / \mathrm{min}\right)$. At normal condition, the peak width is less than $1{ }^{\circ} \mathrm{C}$. All materials exhibit reversible solid-liquid transitions at certain

${ }^{\text {a)} E l e c t r o n i c ~ m a i l: ~ m i n g s u @ m a i l . u c f . e d u . ~}$ temperatures. The melting temperatures depend on atomic numbers (pure metals) and compositions (alloys) if the diameter of PCM materials is larger than critical sizes, where surface atoms play an important role in decreasing melting temperatures. ${ }^{17} \mathrm{~A}$ variety of alloys melt in the operating range of thermal analysis equipment $\left(100-700{ }^{\circ} \mathrm{C}\right),{ }^{18}$ and PCM can be encapsulated inside shells to prevent the changes in compositions. ${ }^{19}$

We have developed a multiplexed highly sensitive method to detect cancer biomarkers (i.e., oligonucleotides), where silica encapsulated phase change nanoparticles of metals is used as thermal barcodes. The silica shells do not melt due to its high melting temperature $\left(1650{ }^{\circ} \mathrm{C}\right)$. The thermal signals are read out by differential scanning calorimetry (DSC), where the melting temperatures and the latent heats of fusion are used to determine the existence and concentrations of biomarkers. The major steps of this method are (1) the immobilization of single strand DNA (ssDNA1) on silica shells of encapsulated PCM nanoparticles; (2) the immobilization of another ssDNA (ssDNA2) on magnetic nanoparticles; (3) the hybridization of target ssDNA at two ends with ssDNA1 and ssDNA2, which will connect encapsulated PCM nanoparticles and magnetic nanoparticles; (4) the magnetic separation of unhybridized target ssDNA and ssDNA1; and (5) the thermal readout of PCM nanoparticles. The sequence of ssDNA1 is $5^{\prime}$-TTA CAA TAA TCC ATT ATT ATT A/3ThioMC3-D-3'; that of target ssDNA is $5^{\prime}$-GAA TTA TTG TAA ACA CAG CAA CCA CAT-3'; and that of ssDNA2 is $5^{\prime}-/ 5$ Biosg/ATG TGG TTG CTG TGT- ${ }^{\prime}$. These sequences are designed randomly and are representative for any genetic biomarkers.

The PCM nanoparticles are synthesized by the thermal decompositions of organometallic precursors. The precursor (bismuth acetate or cyclopentadienyl indium) is dissolved in ethylene glycol in the presence of polyvinylpyrrolidone (PVP). ${ }^{20,21}$ Taking a bismuth nanoparticle as an example, $0.75 \mathrm{mmol}$ bismuth acetate is added into $20 \mathrm{ml}$ ethylene glycol with $0.2 \mathrm{~g}$ PVP. The mixture is heated to $200{ }^{\circ} \mathrm{C}$ in 

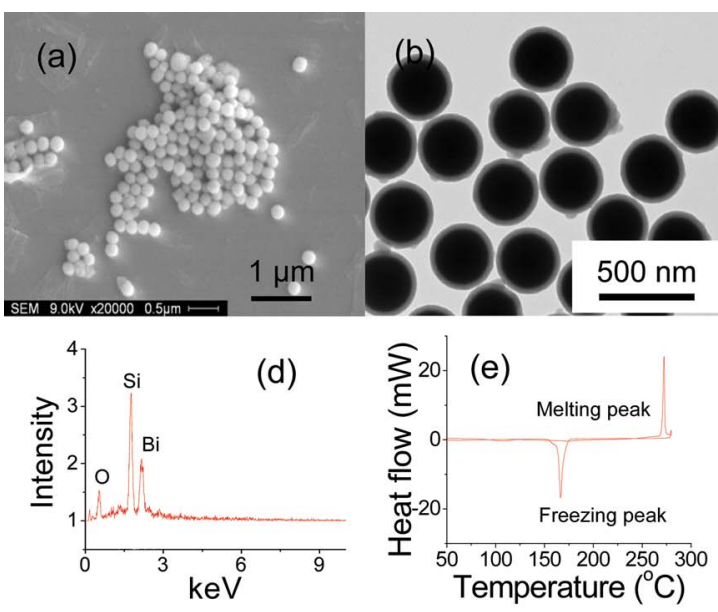

(c)

nitrogen. The reaction is quenched after $20 \mathrm{~min}$ by pouring the mixture into $200 \mathrm{ml}$ ethanol precooled to $0{ }^{\circ} \mathrm{C}$. After centrifuging and washing the nanoparticles, the thermal decomposition of tetraethoxysilane (TEOS) is used to generate silica, where $3.5 \mathrm{ml}$ ammonia hydroxide $(28 \%)$ and $0.2 \mathrm{ml}$ TEOS are added to $50 \mathrm{ml}$ ethanol that contains $50 \mathrm{mg}$ suspended nanoparticles. ${ }^{22}$ The mixture is sonicated at $70{ }^{\circ} \mathrm{C}$ for $1 \mathrm{~h}$, followed by centrifuging and washing by ethanol. Figure 1(a) is a scanning electron microscopy (SEM) image of bismuth nanoparticles. These nanoparticles are coated with $\sim 20 \mathrm{~nm}$ silica as shown in the transmission electron micrograph (TEM) [Fig. 1(b)]. The nanoparticles at room temperature have a crystalline structure as shown in selected area electron diffraction (SAED) pattern [Fig. 1(c)]. Energy dispersive $\mathrm{x}$-ray (EDX) analysis confirms the compositions of core and shell materials [Fig. 1(d)], where the signals of silicon and bismuth can be seen clearly. The solid to liquid phase changes are studied by using DSC, which is operated from 50 to $300{ }^{\circ} \mathrm{C}$ at a ramp rate of $2{ }^{\circ} \mathrm{C} / \mathrm{min}$. The DSC curves of encapsulated bismuth and indium nanoparticles show single peak with full width at half height of less than $1{ }^{\circ} \mathrm{C}$ [Figs. 1(e) and 1(f)] where the two peaks in each figure correspond to the melting and freezing of PCM cores as temperature increases and decreases. Although nanoparticles with diameter smaller than the critical diameter $(10 \mathrm{~nm})$ melt at lower temperatures, encapsulated nanoparticles made here have similar melting temperature as bulk materials due to their larger diameters. ${ }^{23}$

The silica shell is first modified with amine-terminated monolayer by adding aminopropyltriethoxysilane to the suspension of encapsulated nanoparticles in toluene. After reacting for $3 \mathrm{~h}$ at room temperature, extra silane molecules are removed by centrifuging. The nanoparticles are resuspended in anhydrous dimethyl sulfoxide that contains $\mathrm{N}$-succinimidyl (4-iodoacetyl) aminobenzoate (SIAB) and incubated to complete the reaction. After removing excess SIAB by centrifuging, nanoparticles are incubated with 3' thiolated ssDNA (ssDNA1) in a buffer $(p \mathrm{H} \mathrm{8.0)}$ at room temperature. ${ }^{24}$ The ssDNA1 is complementary to one end of the target ssDNA. In order to modify magnetic nanoparticles with separating ssDNA (ssDNA2), which is complementary to the other end of the target ssDNA, avidin is conjugated with polyacrylic-acid-coated iron oxide nanoparticles $(10 \mathrm{~nm}$ diameter). The suspension of avidin-modified magnetic nanoparticles is incubated with biotinylated oligonucleotides in a buffered solution $(p \mathrm{H} 7)$, and unbound ssDNA2 is re- moved by dialysis. The hybridization is done by incubating target ssDNA with excess amounts of ssDNA1 modified PCM nanoparticles, and ssDNA2 modified magnetic nanoparticles in a buffer solution $(p \mathrm{H} 7)$ at $37^{\circ} \mathrm{C}$. After hybridization, the encapsulated PCM nanoparticles with unhybridized ssDNA1 are removed using $\mathrm{NdFeB}$ magnet, and the aggregates formed by the PCM nanoparticles and magnetic particles are transferred into aluminum pans and analyzed using a Perkin-Elmer DSC (DSC 7).

The melting peaks of encapsulated PCM nanoparticles are used for biomarker detections. For encapsulated indium nanoparticles, the width of the melting peak becomes smaller as the ramp rate of temperature decreases [Fig. 2(a)]. A linear relation exists between the ramp rate and the peak width [Fig. 2(b)], where the smallest peak width among the studied ramp rates is $\sim 0.6{ }^{\circ} \mathrm{C}$. The peak areas change with the ramp rates, probably due to the heat loss during heating process. In the case of encapsulated bismuth nanoparticles, the peak widths also depend on the ramp rate [Fig. 2(c)], and the relation between the ramp rate and the peak width is also linear [Fig. 2(d)]. The similarity in both cases suggests that the peak width is mostly determined by the temperature ramp rate. At the peak width of $0.6{ }^{\circ} \mathrm{C}$, the number of thermal barcodes could reach 1000 from 100 to $700{ }^{\circ} \mathrm{C}$. Figure 2(e) shows the melting curves of four aggregates of encapsulated bismuth nanoparticles and iron oxide nanoparticles formed after DNA hybridization. Although iron oxide does not melt during the thermal scan (melting temperature over $1000{ }^{\circ} \mathrm{C}$ ), it can change the heat transfer into PCM nanoparticles because of its low thermal conductivity, thus the melting peaks become asymmetric. As a result, the melting peaks shift toward high temperature side slight, especially at low target ssDNA concentrations, where the ratio of PCM nanoparticles to magnetic nanoparticles is low. The area of melting peak is proportional to the mass of PCM nanoparticles, and can be obtained by integrating the heat flow over the melting range. A linear relation exists between the heat fluxes and the target ssDNA concentrations [Fig. 2(f)], from which the lowest detectable concentration of target ssDNA is $8 \mathrm{nM}$. The detection sensitivity depends on the latent heat of PCM and the diameter of PCM nanoparticles, as well as the grafting density of ssDNA on PCM nanoparticles. For encapsulated nanoparticles of bismuth and indium, the areas of melting peaks are different even if the masses and the diameters of these nanoparticles are close to each other. Lastly, the encapsulated indium and bismuth nanoparticles are mixed at 

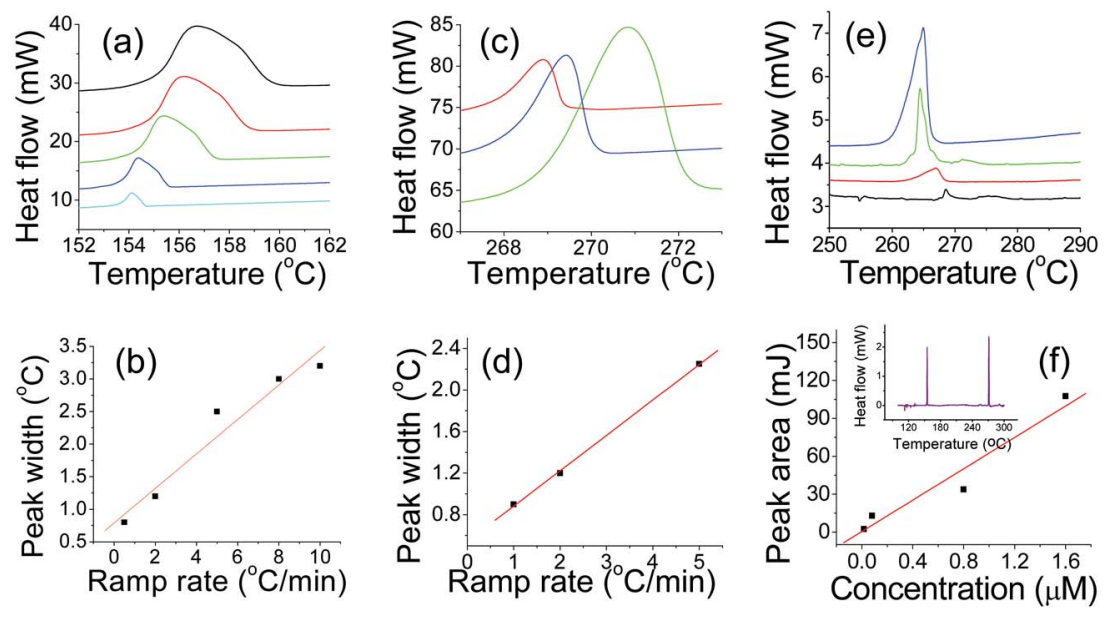

FIG. 2. (Color online) (a) The melting peaks (b) and the relation of peak width and temperature ramp rate for encapsulated indium nanoparticles. (c) The melting peaks and (d) the relation of peak width and the ramp rate for encapsulated bismuth nanoparticles. (e) The melting peaks of encapsulated bismuth nanoparticles after the hybridization with target ssDNA. (f) The relation between peak area and the concentrations of target ssDNA (inset) and the DSC curve of encapsulated indium and bismuth nanoparticles collected in the same detection process. roughly the same concentrations, modified with ssDNA1, and used to detect target ssDNAs. The DSC curve shows two peaks with melting temperatures at 155 and $271{ }^{\circ} \mathrm{C}$, which correspond to the melting peaks of indium and bismuth, respectively. The peak area (i.e., heat flux) of indium is smaller than that of bismuth because the latent heat of indium ( 28 $\mathrm{J} / \mathrm{g})$ is smaller than that of bismuth $(52 \mathrm{~J} / \mathrm{g})$. The ratio of integrated peak area $(0.61)$ is close to the ratio of latent heats of indium and bismuth (0.54), meaning the chances of ssDNA immobilizations, and hybridizations on these two types of nanoparticles are close to each other.

The minimal detectable heat flux in the commercial DSC is determined by its root mean square noise $(0.2 \mu \mathrm{W})$, which corresponds to an energy flow of $0.2 \mu \mathrm{J}$ for $1{ }^{\circ} \mathrm{C}$ wide peak at a ramp rate of $1{ }^{\circ} \mathrm{C} / \mathrm{s}$. If bismuth nanoparticles of $200 \mathrm{~nm}$ diameter are used, the number of nanoparticles that can adsorb $0.2 \mu \mathrm{J}$ heat is $9 \times 10^{4}$. Providing one DNA double helix is formed on each nanoparticle in a $1 \mathrm{ml}$ solution, the accordant concentration of target ssDNA is 1.5 $\times 10^{-16} \mathrm{M}$ or $0.15 \mathrm{fM}$. The current detection limit is not as good as the theoretical one, probably because thousand of target ssDNA are attached on each nanoparticle. The detection sensitivity can be enhanced by using larger diameter particles, materials with large latent heats of fusion, or reducing the grafting density. Microcalorimetry can measure the formation enthalpy of DNA duplex, ${ }^{25}$ but it cannot detect genetic biomarkers due to the sequence-independent enthalpy change. The proposed method works for turbid samples, and is immune to colored molecules, salts, or conductive materials, which do not change phase in the operating range. The interferences from organic carbon can be excluded by preheating samples. The detection capacity and analysis time can be optimized by changing ramp rate. In the case of fast scan, the temperature ramp rate can be $50{ }^{\circ} \mathrm{C} / \mathrm{min}$, and an entire thermal scan from room temperature up to $1000{ }^{\circ} \mathrm{C}$ takes only $20 \mathrm{~min}$. Due to wide ranges of material choices, a one-to-one correspondence can be established between the melting temperatures of PCM nanoparticles and types of biomarkers, allowing easy readout of thermal signals. Lastly, detecting biomarkers in thermal space provides an alternative that is independent of the optical, electric, magnetic, and electrochemical properties of nanoparticles. The large thermal scan range, sharp melting peak, large latent heat, and diverse material choice pave the way to detect multiple low concentration noneffective biomarkers.

This project was supported by the National Science Foundation 0828466, Air Force Research Laboratory, James and Esther King biomedical program of the Florida Department of Health, and the Petroleum Research Fund of American Chemical Society. We appreciate Professor Louis Chow for directing us toward thermal management materials, and Santimukul Santra for surface modification of magnetic nanoparticles.

${ }^{1}$ M. Ferrari, Nat. Rev. Cancer 5, 161 (2005).

${ }^{2}$ K. K. Jain, Curr. Opin. Mol. Ther. 9, 563 (2007).

${ }^{3}$ N. L. Rosi and C. A. Mirkin, Chem. Rev. (Washington, D.C.) 105, 1547 (2005).

${ }^{4}$ J. M. Nam, C. S. Thaxton, and C. A. Mirkin, Science 301, 1884 (2003).

${ }^{5}$ V. Mani, B. V. Chikkaveeraiah, V. Patel, J. S. Gutkind, and J. F. Rusling, ACS Nano 3, 585 (2009).

${ }^{6}$ R. Elghanian, J. J. Storhoff, R. C. Mucic, R. L. Letsinger, and C. A. Mirkin, Science 277, 1078 (1997).

${ }^{7}$ M. Su, S.-Y. Li, and V. P. Dravid, Appl. Phys. Lett. 82, 3562 (2003)

${ }^{8}$ L. Josephson, J. M. Perez, and R. Weissleder, Angew. Chem., Int. Ed. 40, 3204 (2001).

${ }^{9}$ S.-J. Park, T. A. Taton, and C. A. Mirkin, Science 295, 1503 (2002).

${ }^{10}$ J. Wang, D. Xu, A.-N. Kawde, and R. Polsky, Anal. Chem. 73, 5576 (2001).

${ }^{11}$ P. Alivisatos, Nat. Biotechnol. 22, 47 (2004).

${ }^{12}$ J. P. Zimmer, S.-W. Kim, S. Ohnishi, E. Tanaka, J. V. Frangioni, and M. G. Bawendi, J. Am. Chem. Soc. 128, 2526 (2006).

${ }^{13}$ K. A. White and N. L. Rosi, Nanomedicine 3, 543 (2008).

${ }^{14}$ M. Han, X. Gao, J. Su, and S. Nie, Nat. Biotechnol. 19, 631 (2001).

${ }^{15}$ Y. W. C. Cao, R. C. Jin, and C. A. Mirkin, Science 297, 1536 (2002).

${ }^{16}$ E. Hisham, E.-D. Hisham, and A.-K. Eman, Ind. Eng. Chem. Res. 43, 5350 (2004)

${ }^{17}$ E. A. Olson, Y. M. Efremov, M. Zhang, Z. Zhang, and L. H. Allen, J. Appl. Phys. 97, 034304 (2005).

${ }^{18}$ B. Zalba, J. M. Marin, L. F. Cabeza, and H. Mehling, Appl. Therm. Eng. 23, 251 (2003).

${ }^{19}$ M. N. A. Hawlader, M. S. Uddin, and H. J. Zhu, Int. J. Energy Res. 26, 159 (2002).

${ }^{20}$ K. Soulantica, A. Maisonnat, M.-C. Fromen, M.-J. Casanove, P. Lecante, and B. Chaudret, Angew. Chem., Int. Ed. 40, 448 (2001).

${ }^{21}$ F. Wang, R. Yang, H. Yu, P. C. Gibbons, and W. E. Buhro, Chem. Mater. 20, 3656 (2008).

${ }^{22}$ V. G. Pol, S. V. Pol, Y. Gofer, J. Calderon-Moreno, and A. Gedanken, J. Mater. Chem. 14, 966 (2004).

${ }^{23}$ K. Dick, T. Dhanasekaran, Z. Zhang, and D. Meisel, J. Am. Chem. Soc. 124, 2312 (2002).

${ }^{24}$ M. J. O’Donnell, K. Tang, H. Koster, C. L. Smith, and C. R. Cantor, Anal. Chem. 69, 2438 (1997).

${ }^{25}$ A. L. Feig, Biopolymers 87, 293 (2007). 